\title{
Design Features and Ultimate Performance of an Ultra-High Resolution Aberration-Corrected, Monochromatized 200 keV FEG-TEM
}

\author{
A.E. Thesen, ${ }^{*}$ M. Matijevic,* G. Benner* \\ * Carl Zeiss NTS GmbH, Carl-Zeiss-Str. 56, 73447 Oberkochen, Germany
}

We firstly present isotropic TEM imaging resolution reaching the $0.8 \AA$ limit on a $200 \mathrm{keV}$ ultrahigh resolution TEM (UHR-TEM) equipped with a monochromator for illumination and a $\mathrm{C}_{\mathrm{s}^{-}}$ corrector for the imaging system. In order to demonstrate the achieved resolution a Young's fringes pattern (shown in figure 1) has been generated from two micrographs recorded at a magnification of 800,000 times and an acquisition time of one second. The magnification has been calibrated using gold lattice reflections.

This ultimate resolution performance is made possible by narrowing the intrinsic energy spread of the Schottky field emission gun (FEG) of $0.8 \mathrm{eV}$ to below $0.2 \mathrm{eV}$ and thus reducing the damping of sub-Angstrom image features by the temporal envelope function significantly. This energy reduction is achieved by using a dispersion-free monochromator of the electrostatic type, which is housed in the Schottky-FEG. [1] A three stage condensor system allows for Köhler illumination even in connection with the monochromator. By incorporating a $\mathrm{C}_{\mathrm{s}}$-corrector, which follows the design of Rose [2] and has been developed by CEOS, the numerical value of the objective lens spherical aberration, $\mathrm{C}_{\mathrm{s}}$, can be continuously adjusted down to zero and even to negative values. Hence, the phase contrast transfer function (PCTF) of the microscope can be tuned by changing $\mathrm{C}_{\mathrm{s}}$ up to the fundamental limit given by temporal damping.

In order to approach the theoretical resolution limit, extreme care has to be taken to eliminate further damping of the PCTF caused by mechanical and electrical instabilities.

Highest mechanical stability has been realized by a novel "hanging-column concept". [3] In this concept the TEM-column is suspended like a pendulum within a highly stable frame, which has been optimized for maximum stiffness while providing the necessary accessibility to the column. (Fig. 2) In this setup eigen-modes of the column have a knot near the objective lens, thus minimizing vibrations at the sample. By placing the center of gravity of the TEM-column just below the suspension point, the column naturally counteracts tendencies to be tilted out of its upright position. Additional mechanical stability is provided by an increased column diameter of $300 \mathrm{~mm}$.

Furthermore the system is equipped with an in-column energy filter of the in second-order corrected $\Omega$-type. [4] This in connection with the monochromatized FEG enables energy electron loss spectroscopy with an energy resolution well below $200 \mathrm{meV}$.

\section{References}

[1] F. Kahl \& H. Rose, Proc. EUREM Brno/Cz, Vol.III (2000), 1459

[2] H. Rose, Optik 34 (1990), 19

[3] E. Essers et al. Microsc. Microanal. 8 (supppl. 2) (2002), 484CD

[4] G. Lang et al., Microsc. Microanal. 8 (suppl. 2) (2002), 586CD

[5] We gratefully thank M. Lentzen and L. Houben, FZ Jülich, for providing the sample. 


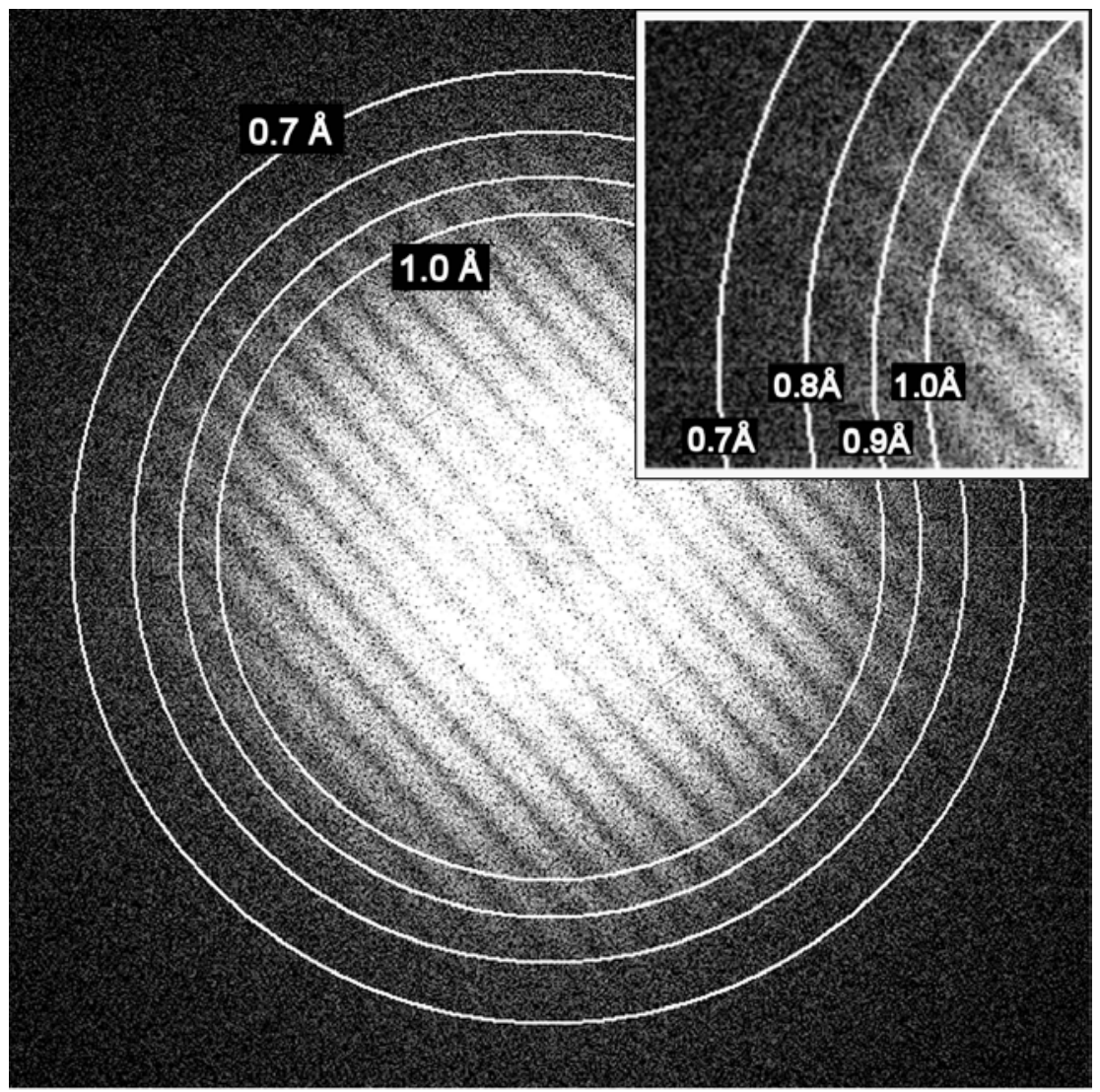

Fig. 1. Young's fringe pattern recorded at $800 \mathrm{k}$ times magnification using a Ta thin film specimen at a residual $\mathrm{C}_{\mathrm{s}}$ value of about $-3 \mu \mathrm{m}$ an energy spread of about $200 \mathrm{meV}$. Four rings calibrated by gold lattice reflections indicate the $1.0 \AA, 0.9 \AA, 0.8 \AA$ and $0.7 \AA$ limits.

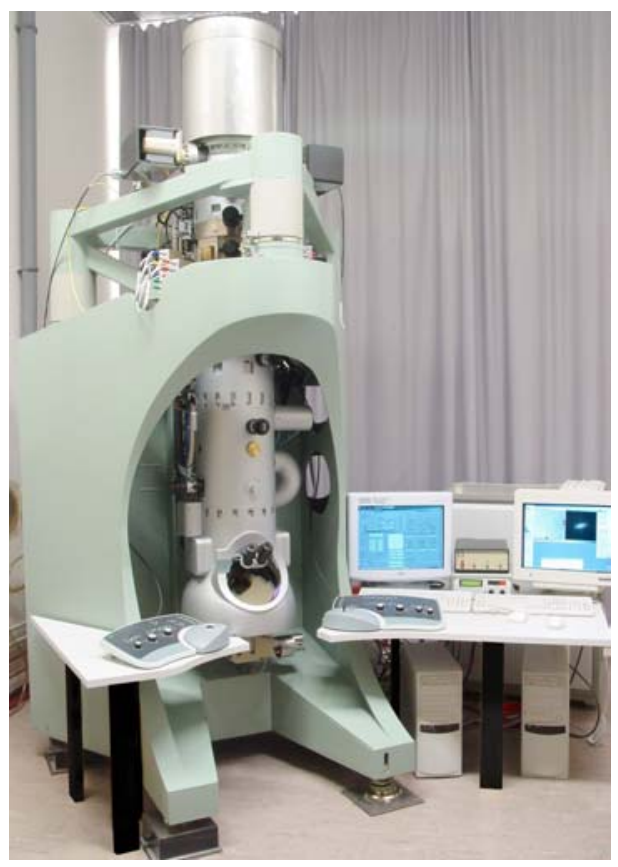

Fig. 2. Photograph of the UHR-TEM with the column suspended in the support structure. 